\title{
A Rare Case of Mucopolysaccharidosis
}

\author{
Ipsita Choudhury • Mona A. Tilak • \\ Arun Kumar Patra
}

Received: 8 March 2013/ Accepted: 16 May 2013/Published online: 8 June 2013

(C) Association of Clinical Biochemists of India 2013

\begin{abstract}
Mucopolysaccharidosis are a group of rare metabolic disorders of the lysosomal storage disease family caused by the absence or malfunctioning of lysosomal enzymes responsible for their breakdown. It encompasses disorders in which undegraded or partly degraded glycosaminoglycans accumulate in the lysosomes of many tissues owing to a deficiency of specific lysosomal enzymes. Here we report a case of a 7 years old child displaying the symptoms of Morquio's disease (Mucopolysaccharidosis type IV). Urine screening tests were performed which gave contrasting results.
\end{abstract}

Keywords Mucopolysaccharidosis ·

Glycosaminoglycans · Lysosomal storage diseases ·

Screening tests

\section{Introduction}

Mucopolysaccharidosis are a group of lysosomal storage disorders caused by deficiency of enzymes catalyzing the stepwise degradation of glycosaminoglycans (GAGs) or mucopolysaccharides. Since lysosomes are involved

I. Choudhury $(\bowtie) \cdot$ M. A. Tilak

Department of Biochemistry, Padmashree Dr. D. Y. Patil

Medical College, Pimpri, Pune, India

e-mail: ipsi_bam@rediffmail.com

A. K. Patra

Department of Anesthesia, AFMC, Pune, India in their degradation, the deficiency in lysosomal enzymes leaves undegraded GAGs to be stored in the lysosomes or excreted in the urine. These diseases are rare with an incidence of around 1 in 1, 32,000 live births. About 9 subtypes of mucopolysaccharidosis are reported (Table 1).

Almost all these subtypes are inherited in the autosomal recessive manner except for the type II with $\mathrm{X}$ linked inheritance. Since mucopolysaccharides are widely distributed in the human tissues; the clinical features may involve virtually all organ systems. Excess of one or more of the GAGs results in numerous physical and mental features, visual and hearing defects, cardiovascular functional impairments, hepatosplenomegaly, and dysostosis multiplex. Symptoms vary in type and severity, depending on which MPS is involved. Severe mental retardation usually is associated with MPS type IH (MPS-IH), MPS type II and MPS type III while types 1S, IV and VI exhibit normal intelligence. Skeletal deformities are particularly severe in types IV and VI $[1,2]$.

\section{Case Report}

A 7 year old male child presented to the pediatrics OPD with the chief complaints of delayed growth and development since birth. He was apparently alright till one year of age, when his mother noticed delayed growth and a protruding thorax. Later the child developed difficulty in walking. The child had normal intelligence and was not associated with seizures or involuntary movements.

The birth history of the child was insignificant. The child was well immunized according to IAP schedule. The developmental history which included the child's gross and fine motor skills was delayed. 
Table 1 Classification of mucopolysaccharidosis

\begin{tabular}{llll}
\hline Number & Eponym & Enzyme & Urinary metabolites \\
\hline MPS I H & Hurlers & $\alpha$-L-Iduronidase & Dermatan sulphate, heparan sulphate \\
MPS I S & Scheie & $\alpha$-L-Iduronidase & Dermatan sulphate, heparan sulphate \\
MPS I H/S & Hurler-Scheie & $\alpha$-L-Iduronidase & Dermatan sulphate, heparan sulphate \\
MPS II (severe) & Hunter (severe) & Iduronate sulphatase & Dermatan sulphate, heparan sulphate \\
MPS II (mild) & Hunter (mild) & Iduronate sulphatase & Dermatan sulphate, heparan sulphate \\
MPS III A & Sanfilippo A & Heparan $N$-sulphatase & Heparan sulphate \\
MPS III B & Sanfilippo B & $\alpha$ - $N$-acetyl glucosaminidase & Heparan sulphate \\
MPS III C & Sanfilippo C & Acetyl CoA: $\alpha$ glucosaminidase acetyl transferase & Heparan sulphate \\
MPS III D & Sanfilippo D & $N$-acetyl glucosamine 6 sulphatase & Heparan sulphate \\
MPS IV A & Morquio A & $N$-acetyl galactosamine-6 sulphatase & Keratan sulphate, chondroitin sulphate \\
MPS IV B & Morquio B & $\beta$-Galactosidase & Keratan sulphate \\
MPS V & - & - & - \\
MPS VI & Maroteaux-Lamy & $N$-Acetyl galactosamine 4 sulphatase & Dermatan sulphate \\
MPS VII & Sly & $\beta$-Glucuronidase & Dermatan sulphate, heparan sulphate \\
MPS VIII & - & - & - \\
MPS IX & Hyaluroni & Hyaluronidase & Hyluron \\
\hline
\end{tabular}

Family History

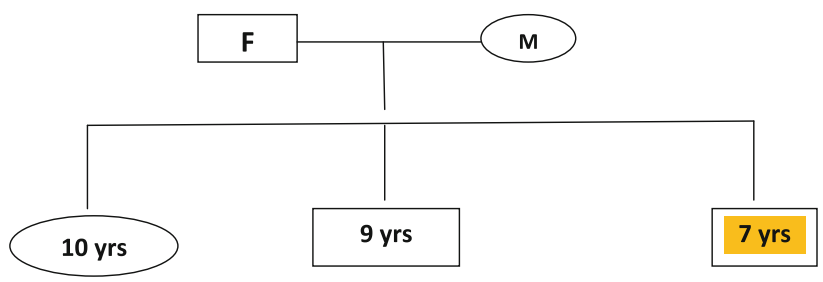

General and Systemic Examination

On examination, patient was conscious, oriented and afebrile. Dysmorphic facial features and macroglossia was evident on the face (Fig. 1). On skeletal examination, the child showed truncal lordosis, scoliosis, odontoid hypoplasia, prominent lateral border of scapula, protruding thorax and waddling gait. Systemic examination of the child was normal with no signs of meningeal irritation ruling out any neurological deficits. On ophthalmological examination typical corneal cloudiness was observed (by slit lamp) (Fig. 2). Pupil was normal and fundus was clear. The laboratory investigations were within normal limits. X'ray spine showed anterior beaking.

\section{Provisional Diagnosis}

As bony abnormalities were more evident than the neurological derangement, the child was provisionally diagnosed to be a case of:

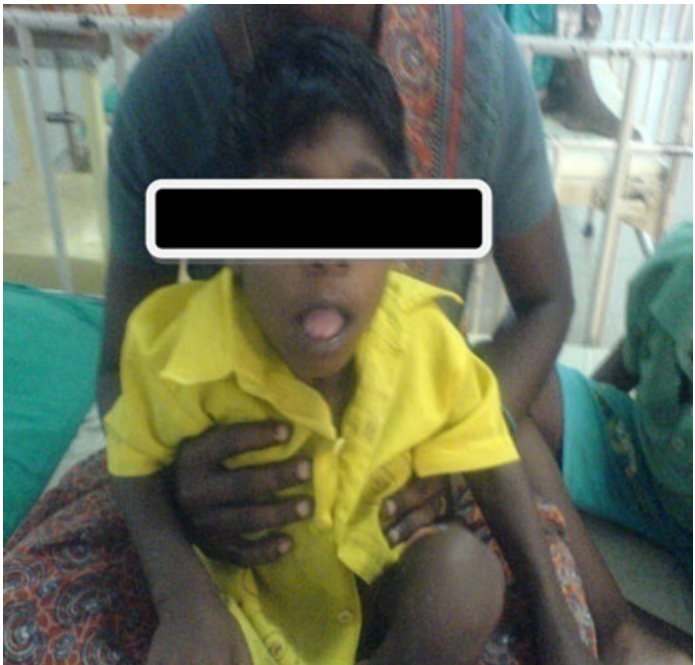

Fig. 1 Child showing dysmorphic facial features

\section{Mucopolysaccharidosis (Type IV) or Morquio's Syndrome}

Lab Studies

1. Toluidine blue (Berry) spot test.

(a) Urine was collected from the patient and his brother for the spot test.

(b) Urine was applied five times to filter paper. (Fig. 3).

(c) Filter papers were stained with toluidine blue, and destained by washing in acetic acid. 


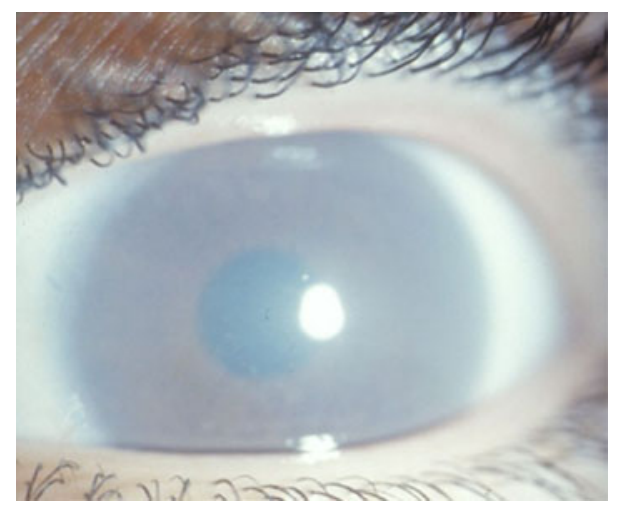

Fig. 2 Clouding of cornea seen through slit lamp examination

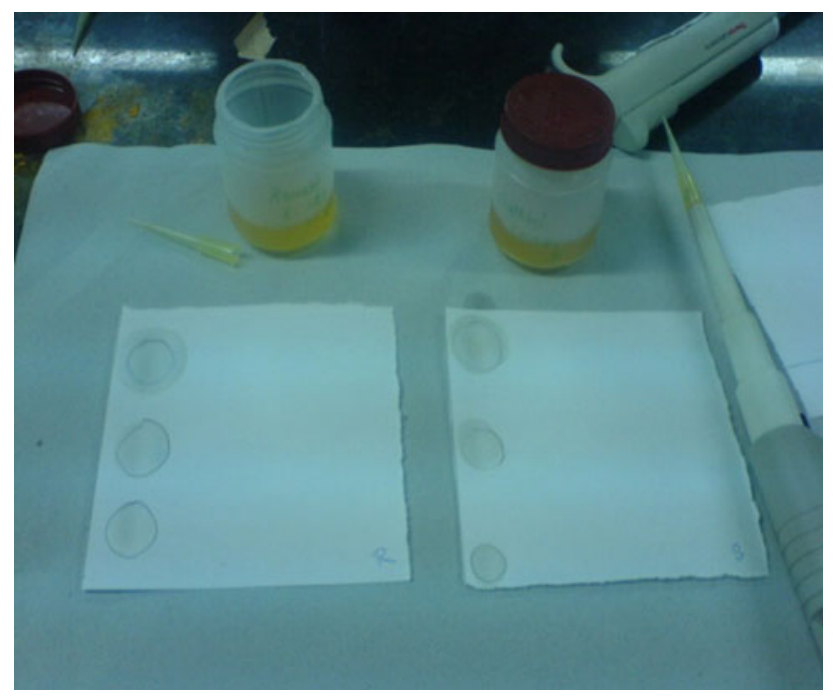

Fig. 3 Urine spotted onto filter papers

(d) No metachromasia was found in the urine sample.

2. Presence of glycosaminoglycans was later confirmed by performing spectrophotometric assay of urine by 1,9 dimethylmethylene blue method.

3. However; specific glycosaminoglycans can only be distinguished by urine electrophoresis techniques to differentiate between the types of mucopolysaccharidoses.

\section{Discussion}

Morquio syndrome is a member of a group of inherited metabolic disorders collectively termed mucopolysaccharidosis (MPSs). It is estimated to occur in 1 in 2, 00,000 live births [3].

Morquio syndrome is divided into two subtypes; Type A $\&$ Type B. Theses subtypes result from the missing or deficient enzymes $N$-acetylgalactosamine 6-sulfatase (Type A) or beta-galactosidase (Type B) needed to break down the keratan sulfate sugar chain. Clinical features are similar in both types but appear milder in Morquio Type B.

Patients with Morquio syndrome usually can be clinically distinguished from patients with other MPSs because they do not have coarse facial features or mental retardation and they have additional skeletal manifestations derived from a unique spondyloepiphyseal dysplasia and ligamentous laxity. These skeletal manifestations include odontoid hypoplasia, a striking short trunk dwarfism, and genu valgus. Compared to other patients with MPS, those with Morquio syndrome tend to have greater spine involvement with scoliosis, kyphosis, and severe gibbus, as well as platyspondyly, rib flaring, pectus carinatum, and ligamentous laxity. Odontoid hypoplasia is the most critical skeletal feature to recognize in any patient with Morquio syndrome. All these features were corroborating with the patients findings.

\section{Pathophysiology}

The most abundant heteropolysaccharides in the body are the glycosaminoglycans (GAGs). These molecules are long unbranched polysaccharides containing a repeating disaccharide unit. The disaccharide units contain either of two modified sugars, $N$-acetylgalactosamine (GalNAc) or $N$-acetylglucosamine (GlcNAc), and a uronic acid (except in keratan sulfate) such as L-glucuronic acid or its 5'epimer, L-iduronic acid [4]. GAGs are highly negatively charged molecules, with extended conformation that imparts high viscosity to the solution making them ideal for lubrication of joints. The specific GAGs of physiological significance are hyaluronic acid, dermatan sulfate, chondroitin sulfate (Fig. 4), heparin, heparan sulfate, and keratan sulfate I \& II. All the GAGs contain sulfate groups except in hyaluronic acid.

The majority of GAGs in the body are linked to core proteins to form proteoglycans. The GAGs extend perpendicularly from the core in a brush-like structure. The linkage of GAGs to the protein core involves a specific trisaccharide composed of two galactose residues and a xylose residue (GAG-GalGalXyl-O-CH -protein (Fig. 5a, b).

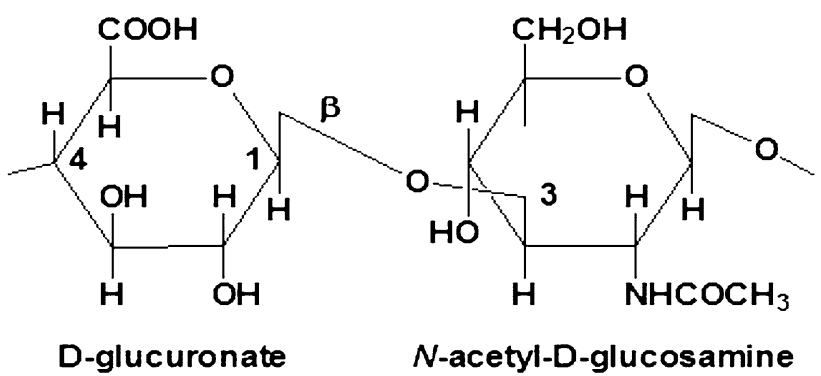

Fig. 4 Structure of chondroitin 4-sulfate 


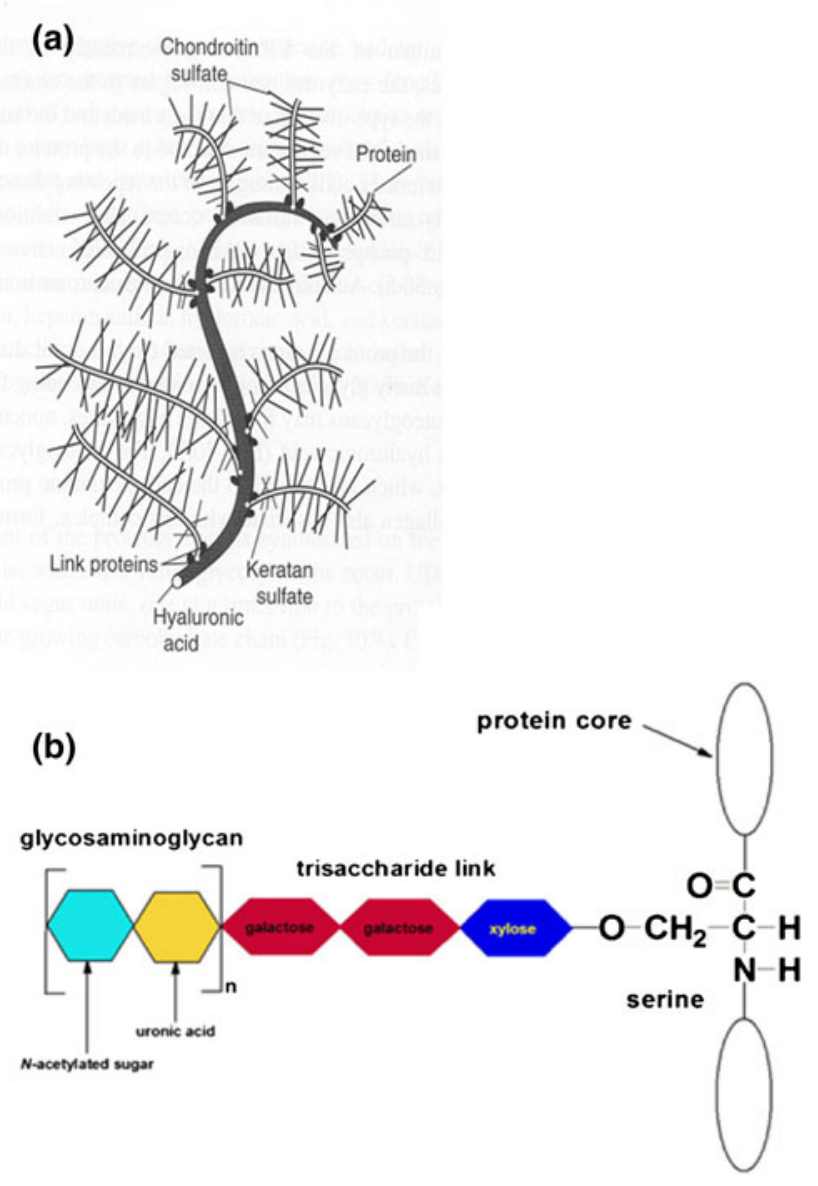

Fig. 5 a Structure of proteoglycan b structure of proteoglycan

GAGs are degraded in a stepwise fashion from the nonreducing end by a series of lysosomal enzymes (exoglycosidases, endoglycosidases and sulfatases). Depending on the specific enzyme deficiency, the catabolism of one or more GAGs may be blocked. Clinical features vary depending on the tissue distribution of the affected substrate and the degree of enzyme deficiency. The enzyme deficiencies are mostly due to the mutations in the genes encoding the specific lysosomal hydrolases.

In Morquio syndrome, the degradation of KS is defective because of deficiency of either $N$-acetyl-galactosamine-6sulfate sulfatase (GALNS gene) in MPS IVA or beta-galactosidase (GLBl gene) in MPS IVB [5, 6]. Defective GALNS also affects the catabolism of chondroitin 6-sulfate.

KS (keratan sulphate) [7] is predominantly found in cartilage and cornea, the major organs affected in Morquio syndrome. Thus in Morquio's, the bone and joint abnormality are more common as compared to the neurological disturbances.

\section{Clinical Features of Morquio [8, 9]}

- Genu valgus

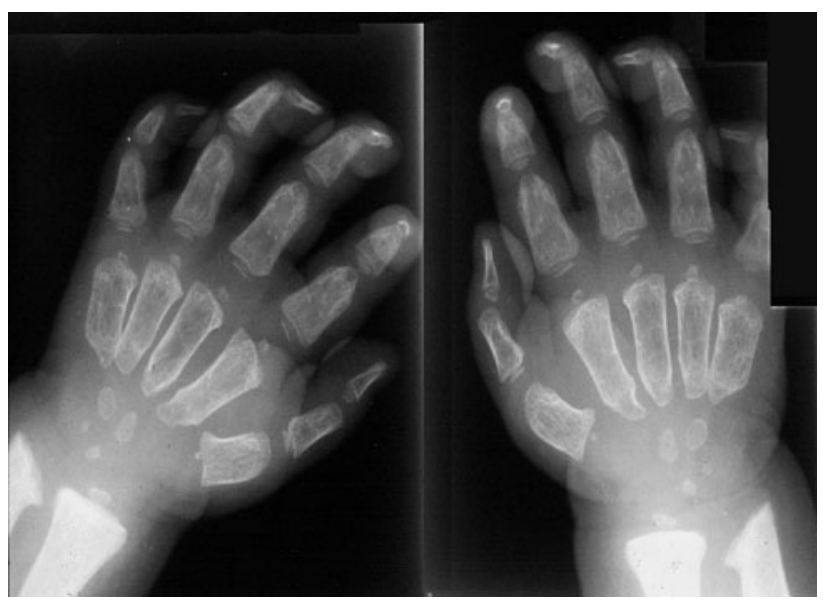

Fig. 6 Metacarpal widening with proximal pointing

- Kyphosis

- Waddling gait

- Dwarfism

- Platyspondylysis

- Odontoid hypoplasia

- Hyperlordosis

- Osteoporosis

- Ulnar deviation

- Widening of metacarpals with proximal pointing (Fig. 6).

Extra skeletal features

- Mild corneal clouding [10]

- Hepatomegaly

- Cardiac valvular lesion

- Small teeth

- Thin enamel

- Frequent caries formation

\section{Differential Diagnosis}

(1) Other disorders of MPS

(2) Spondyloepiphyseal dysplasia

(3) Multiple epiphyseal dysplasia.

\section{Investigations}

- Urine spot tests Urine spot tests are forerunners for screening mucopolysaccharides (GAG) [11]. However; these tests are associated with false-positive and falsenegative results. In Morquio syndrome, mildly affected patients do not always excrete KS fragments. This test showed negative results in our patient which may be explained by the aforesaid facts. 
- Semiquantification of urinary GAG can be obtained by spectrophotometric assays with dimethylmethylene blue dye.

- Heparan, keratan, and dermatan sulfate can be distinguished by separation techniques like electrophoresis or thin layer chromatography to narrow the differential among the mucopolysaccharidosis (MPSs) [12].

Other investigations which can be done are:

- Enzyme assays in leucocytes, fibroblasts or in serum $[13,14]$.

- Tissue biopsy of the affected area and identification of GAG by electrophoresis.

- Prenatal diagnosis in cell free amniotic fluid or by chorionic villus biopsy.

- Detection of mutations in the GALNS and GLB1 genes can facilitate carrier testing if the family desires [15].

- A new enzyme-linked immunoassay (ELISA) technique has recently been shown to accurately quantify keratan sulfate in urine and blood in patients with MPS type IVA.

Imaging Studies a) A full skeletal survey should be obtained in a patient thought to have MPS.

b) CT scanning or MRI of the brain stem and cervical spine should be performed to evaluate odontoid hypoplasia and cord compression. The authors recommend additional CSF flow studies in flexion and extension in patients older than 5 years.

Other Tests An ophthalmology examination with slit lamp should be performed at the time of initial evaluation to look for corneal clouding.

Histologic Findings The lysosomes of patients with MPS are engorged with unmetabolized GAG. These appear as vacuoles or inclusion bodies in cells such as lymphocytes, hepatocytes, corneal epithelium, and neurons [16].

\section{Treatment}

Genetic counseling should be provided to patients with newly diagnosed MPS and their families. Because this is an autosomal recessive disorder, a $25 \%$ risk for the parents to have another child with Morquio syndrome exists in each pregnancy.

\section{Medical Care}

- Only palliative measures are currently available for treatment of patients with Morquio syndrome.
- Potential strategies for treatment of patients with the other mucopolysaccharidoses (MPSs), which are currently at different levels of development, include enzyme replacement, gene therapy, and allogenic bone marrow transplantation in which engrafted cells provide the normal enzyme.

- Currently, enzyme replacement therapy is available commercially for MPS type I (Laronidase) [17] and MPS type II (Idursulfase) [18].

- One of the latest experimental drug for Morquio A syndrome is an enzyme replacement of GALNS called $B M N 110$ which is still under clinical trial [19].

\section{Surgical Care}

- Because all patients with Morquio syndrome have odontoid hypoplasia that can lead to atlantoaxial subluxation, many physicians recommend cervical spine fusion for all of their affected patients

- Other potential operations for patients with Morquio syndrome include femoral osteotomies and corrective knee surgery for genu valgus deformity. Total joint replacement of hips and/or knees may be necessary. The early use of a back brace may delay or prevent surgical intervention for scoliosis.

- Corneal grafting for progressive corneal haziness has been reported with variable recurrence success.

\section{References}

1. Muenzer J, Neufeld EF. Mucopolysaccharidosis. In: Scriver CR, et al., editors. The metabolic basis of inherited disease. 6th ed. New York: Mc Graw hill information services Co; 1989.

2. Sly WS. The mucopolysaccharidosis. In: Bondy PK, LE Rosenberg, editors. Metabolic control and disease. 8th ed. Philadelphia: WB Saunders Co; 1980.

3. National Institute of Neurological Disorders and Stroke. Mucolipidoses Fact Sheet. Office of Communications and Public Liaison. Bethesda, MD; Publication No. 03-5115, 2007.

4. Gandhi NS, Mancera RL. The structure of glycosaminoglycans and their interactions with proteins. Chem Biol Drug Des. 2008;72(6):455-82.

5. Suzuki Y, Oshima A, Nanba E. Beta-galactosidase deficiency (beta-gGalactosidosis): GM1 gangliosidosis and morquio B disease. In: Scriver CR, Beaudet AL, Sly WS, editors. The metabolic and molecular basis of inherieted disease. 8th ed. New York: McGraw-Hill, Inc; 2001. p. 3775-809.

6. Callahan JW. Molecular basis of GM1 gangliosidosis and Morquio disease, type B. Structure-function studies of lysosomal beta-galactosidase and the non-lysosomal beta-galactosidase-like protein. Biochim Biophys Acta. 1999;1455(2-3):85-103.

7. Funderburgh JL. Keratan sulfate: structure, biosynthesis, and function. Glycobiology. 2000;10(10):951-8.

8. Holzgreve W, Grobe H, von Figura K, et al. Morquio syndrome: clinical findings in 11 patients with MPS IVA and 2 patients with MPS IVB. Hum Genet. 1981;57(4):360-5. 
9. Resnick D. Osteochondrodysplasias, dysostoses, chromosomal aberrations, mucopolysaccharidoses, mucolipidoses and other skeletal dysplasias. Diagnosis of bone and joint disorders, vol. 5. Philadelphia: WB Saunders Co; 1988. p. 3501-7.

10. Ashworth JL, Biswas S, Wraith E, Lloyd IC. Mucopolysaccharidoses and the eye. Surv Ophthalmol. 2006;51(1):1-17.

11. Tomatsu S, Okamura K, Taketani T, et al. Development and testing of new screening method for keratan sulfate in mucopolysaccharidosis IVA. Pediatr Res. 2004;55(4):592-7.

12. Rattenbury JM, Worthy E, Allen JC. Screening tests for glycosaminoglycans in urine:experience from regional interlaboratory surveys. J Clin Pathol. 1988;41:936-9.

13. Ullrich K. Screening for lysosomal disorders. Eur J Pediatr. 1994;153(7 Suppl 1):S38-43.

14. Yuen M, Fensom AH. Diagnosis of classical Morquio's disease: $\mathrm{N}$-acetylgalactosamine-6-sulphate sulphatase activity in cultured fibroblasts, leukocytes, amniotic cells and chorionic villi. J Inherit Metab Dis. 1985;8(2):80-6.
15. Tomatsu S, Montano AM, Nishioka T, et al. Mutation and polymorphism spectrum of the GALNS gene in mucopolysaccharidosis IVA (Morquio A). Hum Mutat. 2005;26(6):500-12.

16. Leslie T, Siddiqui MA, Aitken DA, et al. Morquio syndrome: electron microscopic findings. Br J Ophthalmol. 2005;89(7): 925-6.

17. Dupont C, Hachem CE, Harchaoui S, et al. Hurler syndrome: early diagnosis and successful enzyme replacement therapy: a new therapeutic approach. Case report. Arch Pediatr. 2008;15(1): 45-9.

18. Garcia AR, DaCosta JM, Pan J, Muenzer J, Lamsa JC. Preclinical dose ranging studies for enzyme replacement therapy with idursulfase in a knock-out mouse model of MPS II. Mol Genet Metab. 2007;91(2):183-90.

19. A Study to Evaluate the Safety, Tolerability and Efficacy of BMN 110 in Subjects With Mucopolysaccharidosis IVA. http:// clinicaltrials.gov/show/NCT00884949. 